\title{
HERA Beam Tail Shaping by Tune Modulation
}

\author{
Christoph Montag
}

BNL, Upton, NY 11973

\begin{abstract}
To study CP violation, the HERA-B experiment uses an internal wire target in the transverse halo of the stored HERA proton beam. Operational experience shows that the resulting interaction rates are extremely sensitive to tiny orbit jitter amplitudes. Various methods have been studied to stabilize these interaction rates by increasing diffusion in the transverse proton beam tails without affecting the luminosity at the electron-proton collider experiments ZEUS and H1. Tune modulation was found to be a promising method for this task. Experiments performed in recent years will be reported.
\end{abstract}

\section{INTRODUCTION}

The electron-proton collider HERA consists of a superconducting $920 \mathrm{GeV}$ proton storage ring and a $27.5 \mathrm{GeV}$ electron storage ring. Beams collide in two of the four interaction regions (IRs), which are equipped with the detectors ZEUS (IR South) and H1 (IR North). In the interaction region East the experiment HERMES with its polarized internal gas target is installed in the electron ring to measure the spin structure of protons and neutrons using the stored polarized HERA electron beam. The HERA-B detector is installed in the straight section West of the HERA proton ring to study CP violation in the B-meson decay $B^{0} \rightarrow J / \psi K_{s}^{0}$, using an internal wire target in the beam halo. To achieve the design interaction rate of $\dot{N}_{\text {interaction }}=40 \mathrm{MHz}$, each bunch crossing has to provide about four interactions. To enable reconstruction of these interactions, a sufficient spatial distribution is required. This is achieved by arranging four target wires around the beam, operated simultaneously. Each of these wires is equipped with a stepping motor to ensure equal contribution of all four wires to the overall interaction rate. These motors allow for accurate target movements in nominal steps of $50 \mathrm{~nm}$. The interaction rate is kept constant by a $10 \mathrm{~Hz}$ wire position feedback which counteracts slow beam orbit variations by target position adjustment.

Observations demonstrate that this slow wire position feedback is not sufficient to ensure the required interaction rate stability. At high interaction rates above some $20 \mathrm{MHz}$ large fluctuations were observed, with relative rms deviations

$$
\sigma_{\text {rel }}=\frac{\sqrt{\left\langle\left(\dot{N}_{\text {interaction }}-\left\langle\dot{N}_{\text {interaction }}\right\rangle\right)^{2}\right\rangle}}{\left\langle\dot{N}_{\text {interaction }}\right\rangle}
$$

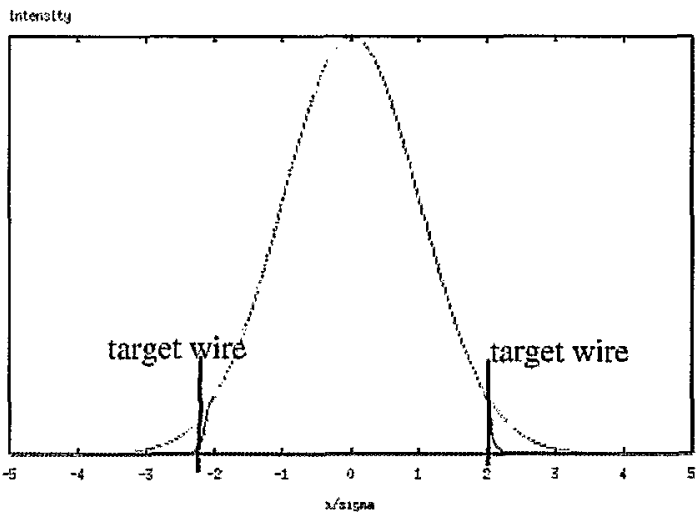

FIGURE 1. Schematic view of the horizontal HERA-B wire target in the halo of the stored proton beam. Due to insufficient diffusion the wires have to move ever closer to the beam core, thus scraping away the halo. The resulting "hard edge" of the modified distribution leads to large interaction rate fiuctuations when the beam orbit varies due to effects such as ground motion.

above $50 \%$. This effect can be explained by lack of diffusion into the transverse beam tails, requiring the eventual positioning of the target wires extremely close to the beam center, typically at 3 to 4 transverse rms beam sizes. As Figure 1 shows, this tight target aperture leads to sharp "edges" of the initially Gaussian distribution, resulting in a high sensitivity of the interaction rate to disturbances such as beam orbit jitter.

To overcome this difficulty, a re-population of the transverse proton beam tails by increased diffusion is required. However, since HERA has to simultaneously provide high luminosity electron-proton collisions at the colliding-beam experiments ZEUS and $\mathrm{H} 1$, this "tail 
shaping" must be performed without any detrimental effects like luminosity degradation or increased background rates. A simple slow transverse blow-up of the beam is therefore not tolerable, and a more sophisticated method has to be chosen.

Tune modulation in connection with a strong nonlinearity, in particular the beam-beam interaction, is well known to result in increased diffusion in the transverse beam tails. The beam core, where the linear part of the beam-beam force dominates, is practically unaffected $[1,2,3,4]$. This is therefore an ideal approach for "tail shaping".

\section{EXPERIMENTAL SET-UP}

The main quadrupoles of the $920 \mathrm{GeV}$ superconducting HERA proton storage ring are connected in series with the main dipole magnets. For fine-adjustment and control of the tunes two families of superconducting quadrupole magnets are installed in each HERA quadrant. The chopper power supplies of these quadrupoles in the quadrant West are modified to provide an additional control input, allowing for tune modulation with frequencies up to about $1.5 \mathrm{kHz}$.

The tune modulation signal is generated by a $\mathrm{PC}$ with DATEL PC-420 wave generator board [5], remotely controllable from the HERA control room. This board generates an arbitrary, continuous, periodic signal that is used as the input signal for the quadrupole chopper power supplies.

The large inductance of the superconducting quadrupole magnets leads to an effective suppression of high frequency modulation signals, while low frequencies of a few Hertz are partially counteracted by the power supplies' internal feedback loops. Figure 2 shows the measured transfer function of this assembly.

\section{EXPERIMENTS}

The effect of tune modulation on the beam halo was studied in two steps. First a single target wire was moved into the beam halo until the interaction rate reached $10 \mathrm{MHz}$. Then the wire was retracted by $100 \mu \mathrm{m}$ and the target postion feedback was turned off. After an initial decay time the interaction rate reached a stable value of about $0.3 \mathrm{MHz}$. When the tune modulation was turned on, the interaction rate increased until a new equilibrium was reached. This equilibrium value was recorded for different tune modulation parameters, as shown in Figure 3 , with

$$
U(t)=\sum_{i} U_{i} \cdot \sin \left(2 \pi f_{i} t\right)
$$

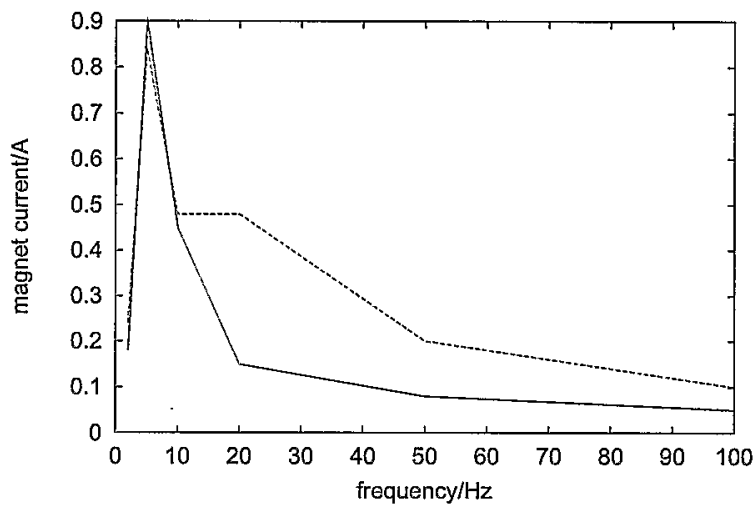

FIGURE 2. Measured AC peak magnet current as function of frequency for an input voltage of $1 \mathrm{~V}$ and for two DC magnet currents of $30 \mathrm{~A}$ (lower) and $60 \mathrm{~A}$ (upper), respectively. The lines are drawn to guide the eye.

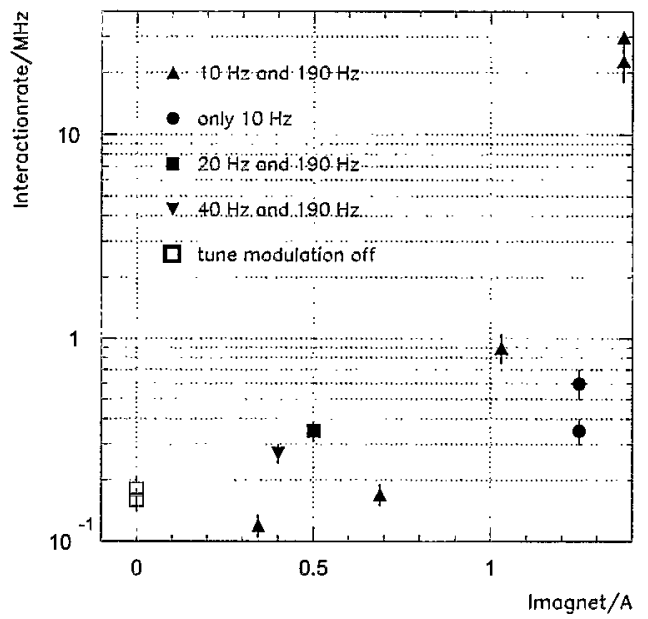

FIGURE 3. Interaction rates with stationary wire for different tune modulation parameters. For each setting, the input signal voltages for each frequency component are identical, but their sum might be varied. Due to the strong frequency dependency of the magnet power supply output current, the resulting total tune modulation amplitude is different for different frequency parameter sets.

being the power supply $\mathrm{AC}$ input voltage. The amplitude of all frequency components $U_{i}$ of the input signal was equal for all measurements, but due to the frequency dependency of the magnet currents the resulting tune modulation depths were not equal.

The maximum interaction rate was achieved by simultaneous application of two modulation frequencies: 
$f_{2}=190 \mathrm{~Hz}$ and $f_{1}=10,20$, or $40 \mathrm{~Hz}$, see Figure 3 . At an $\mathrm{AC}$ magnet current of $1.37 \mathrm{~A}$, an interaction rate of 23 resp. $30 \mathrm{MHz}$ could be achieved using $f_{1}=10 \mathrm{~Hz}$ and $f_{2}=190 \mathrm{~Hz}$. When the $190 \mathrm{~Hz}$ component was turned off, the resulting interaction rate dropped significantly to 0.35 resp. $0.6 \mathrm{~Hz}$, though the relative reduction in the total AC peak magnet current to $1.25 \mathrm{~A}$ was much smaller. With the $190 \mathrm{~Hz}$ component turned on again and reduced amplitudes of both components, the resulting $0.9 \mathrm{MHz}$ interaction rate was significantly larger than with $10 \mathrm{~Hz}$ only though the total resulting tune modulation depth was reduced. Replacing the $10 \mathrm{~Hz}$ frequency component by a 20 or $40 \mathrm{~Hz}$ component while keeping the $190 \mathrm{~Hz}$ component constant shows a clear correlation between total tune modulation amplitude and resulting HERA-B interaction rate, see Figure 3. But since these frequencies are already strongly suppressed by the large magnet inductance the maximum achievable interaction rate is still much smaller than in the case with the $10 \mathrm{~Hz}$ component.

In a later dedicated HERA run the effect of tune modulation on HERA-B interaction rate stability was studied. For this study the wire position feedback was switched on and a single target wire was inserted into the beam halo until a stable interaction rate of $20 \mathrm{MHz}$ was reached. To avoid interference effects with the $10 \mathrm{~Hz}$ wire position feedback, the lower frequency $f_{1}$ was set to $8 \mathrm{~Hz}$ resp. $12 \mathrm{~Hz}$ during these experiments.

With the lower modulation frequency set to $f_{1}=12 \mathrm{~Hz}$, the effect of the second frequency component $f_{2}$ was studied in steps of $100 \mathrm{~Hz}$ for input modulation signal amplitudes of $0.5 \mathrm{~V}$ and $1.0 \mathrm{~V}$, respectively. As an example, Figure 4 shows the effect on the interaction rate stability for one configuration. Figure 5 depicts the resulting relative rms interaction rate width $\sigma_{\text {rel }}$ as a function of $f_{2}$ for different tune modulation depths.

In contrast to the previous measurement at a fixed wire position, the resulting interaction rate stability does not correlate with the second frequency $f_{2}$. The rate stability exhibits a strong dependency on the amplitude of the input signal, and thus on the resulting modulation depth. This behavior was also observed when the $12 \mathrm{~Hz}$ component was replaced by an $8 \mathrm{~Hz}$ signal.

With the tune modulation switched on, the interaction rate per single proton bunch is clearly correlated to the intensity of the corresponding electron bunch, see Figure 6. This indicates that the observed stabilization effect is indeed dominated by tune modulation in combination with the beam-beam interaction, rather than a simple orbit effect due to orbit offsets in the modulated quadrupoles.

During these experiments the specific luminosity at the two colliding beam experiments ZEUS and $\mathrm{H} 1$ was recorded. As shown in Figure 7, these tune modulation

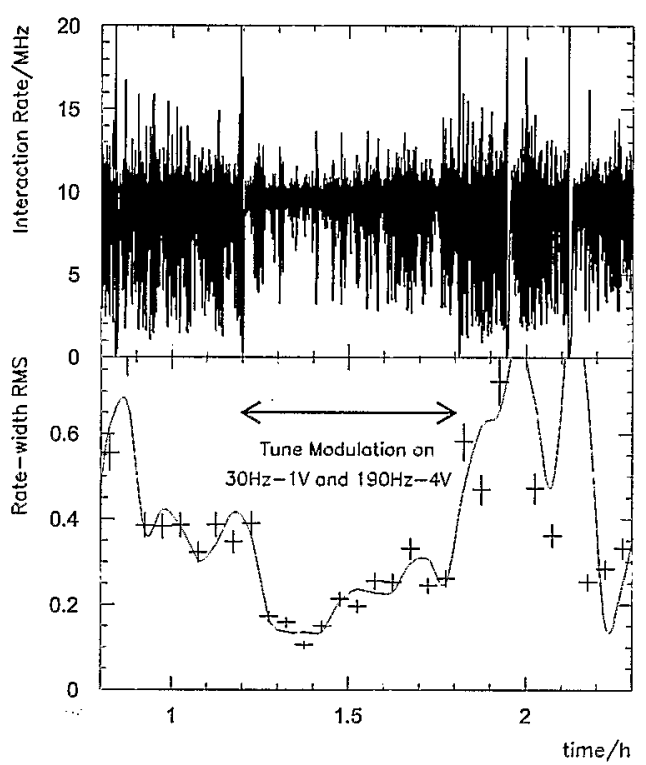

FIGURE 4. Example of the effect of tune modulation at $f_{1}=30 \mathrm{~Hz}, f_{2}=190 \mathrm{~Hz}$ on rate stability. When the modulation is turned on, the relative width of the interaction rate drops from some $50 \%$ to roughly $25 \%$, and increases immediately to the previous level after the tune modulation turned off.

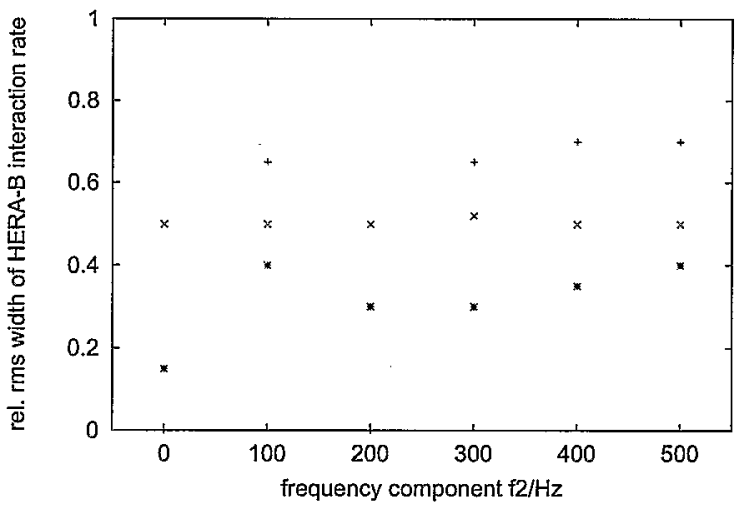

FIGURE 5. Interaction rate stability for different tune modulation frequencies $f_{2}$ and three different amplitudes $(+: 0.5 \mathrm{~V}$, $x: 1.0 \mathrm{~V}, *: 2.0 \mathrm{~V})$. The case without a second frequency component, i. e. $12 \mathrm{~Hz}$ only, is indicated as $f_{2}=0 \mathrm{~Hz}$.

studies did not result in any decrease of specific luminosity. Furthermore, no significant experimental background increase was observed. 

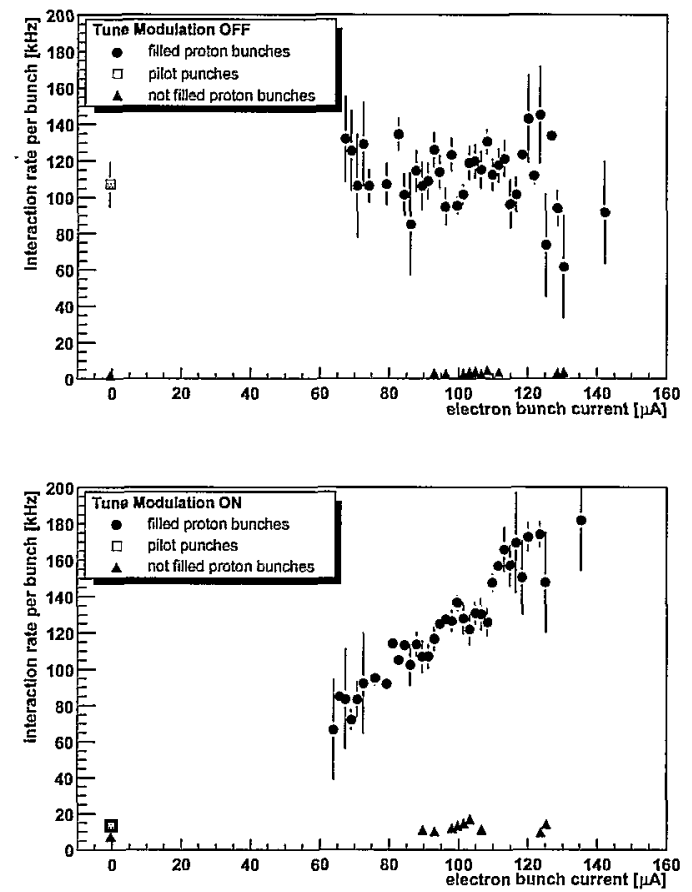

FIGURE 6. HERA-B interaction rate per bunch vs. bunch current of the corresponding electron bunch. Note that the last two proton bunches ("pilot bunches") in each 60 bunch train have no counterpart in the electron beam.

\section{CONCLUSION}

Interaction rate stabilization at the internal halo wire target of the HERA-B experiment by means of tune modulation has been successfully demonstrated. Since this method does not result in any detrimental effects such as luminosity degradation or increased background rates at the colliding beam experiments ZEUS and $\mathrm{H1}$, it has become a standard procedure in regular HERA runs.

\section{REFERENCES}

1. S. Peggs, Nonlinear Dynamics Experiments, in: A. W. Chao, M. Tigner (eds.), Handbook of Accelerator Physics and Engineering, World Scientific, Singapore, 1999

2. L. Evans, The Beam-Beam Interaction, Proc. CERN Accelerator School, CERN 84-15

3. O.S. Bruening, Emittance Growth in Proton Storage Rings due to the Combined Effect of Non-Linear Fields and Modulation Effects with more than one Frequency, DESY HERA 93-10

4. O. S. Bruening, An Analysis of the Long-Term Stability of the Particle Dynamics in Hadron Storage Rings, DESY

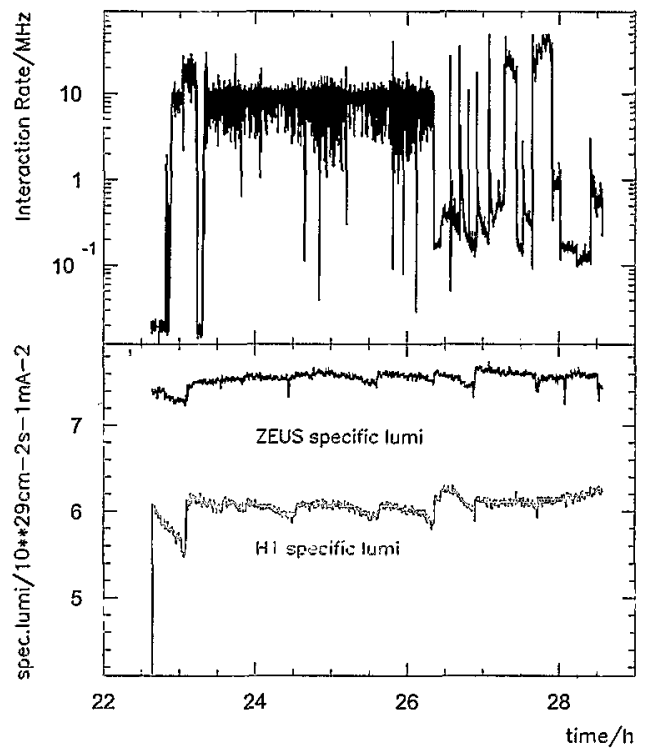

FIGURE 7. HERA-B interaction rate (top) and specific luminosity (bottom) during a dedicated tune modulation study run. The specific luminosity tends to deteriorate due to slow orbit drifts, but recovers completely to the initial value after orbit correction is applied.

\section{4-085}

5. DATEL PC-420 User Manual

6. I. Ludwig, private communication 